\title{
Functional assessment of geriatric patients in regard to health-related quality of life (HRQoL)
}

\author{
This article was published in the following Dove Press journal: \\ Clinical Interventions in Aging \\ 19 December 2014 \\ Number of times this article has been viewed
}

\author{
Marta Muszalik' \\ Tomasz Kornatowski \\ Halina Zielińska- \\ Więczkowska ${ }^{3}$ \\ Kornelia Kędziora- \\ Kornatowska' \\ Ate Dijkstra ${ }^{4}$
}

'Clinic of Geriatrics, Nicolaus Copernicus University Collegium Medicum in Bydgoszcz, Bydgoszcz, Poland; ${ }^{2}$ Department of Preventive Medicine and Environmental Health, Nicolaus Copernicus University Collegium Medicum in Bydgoszcz, Bydgoszcz, Poland; ${ }^{3}$ Department of Pedagogy and Nursing Didactics, Nicolaus Copernicus University Collegium Medicum in Bydgoszcz, Bydgoszcz, Poland; ${ }^{4}$ Research group iHuman, NHL University for Applied Sciences, Leeuwarden, the Netherlands
Correspondence: Marta Muszalik

Clinic of Geriatrics, Nicolaus Copernicus University Collegium Medicum in

Bydgoszcz, ul Marii Curie-Skłodowskiej 9, Bydgoszcz, Poland

Tel +48525854900

Fax +48525854921

Email muszalik@cm.umk.pl
Purpose: The main aim of our research was to evaluate general health, functioning, and performance parameters, as well as care problems of Geriatric Clinic inpatients in relation to deficits in fulfilling needs. The assessment of health-related quality of life was also performed.

Patients and methods: The research subjects were patients attending the Clinic of Geriatrics: 149 women and 78 men; 227 persons in total. The research was carried out using a diagnostic poll method, with the application of the Activities of Daily Living questionnaire of assessment of daily efficiency on the basis of the Katz index, the Instrumental Activities of Daily Living questionnaire, the Care Dependency Scale used to measure the level of care dependency and human needs, and the Nottingham Health Profile scale.

Results: The results showed that the majority of respondents achieved high and medium levels of functional capability. The main problems associated with the fulfillment of needs were difficulties with the adoption of appropriate body posture, movement restrictions, and problems related to participating in unassisted leisure activities outside the home. The general deficit in fulfilling the needs of the patients was low. The most significant problems were related to sleep disorders, restrictions in freedom of movement, loss of vital energy, and ailments resulting in the observable presence of pain.

Conclusion: Good daily functioning of elderly patients significantly depended on their intellectual and mental efficiency. Elderly patients require a comprehensive, holistic approach to a variety of problems that occur with aging.

Keywords: daily activity, elderly people, human needs

\section{Introduction}

The aging population of Europe and worldwide means that there are increasing numbers of people being affected by health problems who are living with reduced functional efficiency. Thus, it is necessary to understand the problems as well as social, psychological, and medical needs of chronically ill patients in order to plan their optimal interdisciplinary care. ${ }^{1}$

Currently, there is worldwide interest in the development of medical research in the field of quality of life (QoL) as affected by health. This can be focused on specific groups of patients selected according to their ailments or in general to describe the population of elderly patients. ${ }^{2-8}$ There is a strong association between health and QoL. This association can be assessed using health-related quality of life (HRQoL) metrics, which include measures of physical, biological, psychological, and social status. ${ }^{9}$

Medical sciences, including nursing, continue to seek methods to assess the functional performance, needs, and QoL of geriatric patients in particular. There are some recent developments, including new research tools for assessing the health needs of patients that can be applied in clinical practice. One example of a psychometric 
tool is the Care Dependency Scale (CDS). The scale was created in the Netherlands in 1994, and has since been continuously developed.$^{10-13}$ The scale was developed from the V Henderson nursing model, based on Maslow's hierarchy of human needs. ${ }^{14}$ This tool measures the patient's needs and his/her care dependency (care profile), and assesses certain ailment risks (risk profile required for effective nursing).

The main aim of our research was to evaluate general health, functioning, and performance parameters, as well as care problems of Geriatric Clinic inpatients in relation to deficits in fulfilling needs. The assessment of HRQoL was also performed.

\section{Materials and methods Participants}

The study included 227 patients aged 60 years or above without serious cognitive impairment. The results of the mini-mental state examination (MMSE) were received from psychological documentation. In the study group, 72 patients (27.3\%) received a normal score MMSE (27-30 points [p]), 83 respondents a score in the range $24-26$ p, while $82(36.1 \%)$ showed a mild degree of dementia (19-23 p). Mild dementia was not an exclusion criterion.

In most cases $(54.6 \%)$, the patients were scheduled to be admitted to hospital, while other patients were admitted in emergency situations (45.4\%). For the majority of patients studied (128 patients; 56.4\%), it was not their first hospitalization. Upon admission, most patients were of average (143; $63 \%)$ or good health $(72 ; 31.7 \%)$, whereas $12(5.3 \%)$ were admitted with a medium-severe condition. The data from the patients was collected between the third and fifth day of their stay, when their health was good enough to answer the questionnaires. The reasons for admission to hospital included: prevalent cardiac diseases $(71 ; 31.3 \%)$, metabolic diseases $(67 ; 29.5 \%)$, respiratory system diseases $(30 ; 13.2 \%)$, and musculoskeletal system diseases $(24 ; 10.6 \%)$. Other patients suffered from cancer, urinary system diseases, or gastrointestinal diseases. The distribution of the duration of chronic diseases in the study group was as follows: $31.3 \%$ patients had suffered for up to 5 years, $43.2 \%$ for 5-15 years, and $25.6 \%$ for $>15$ years.

\section{Measurements and procedure}

The study included a short interview to gather social and demographic data. All patients were assessed for functional performance parameters using standardized geriatric practice $^{15}$ research tools: ADL scale (Activities of Daily Living), ${ }^{16}$ IADL scale (Instrumental Activities of Daily Living), ${ }^{17}$ and a questionnaire assessing human needs CDS. The ADL is used in practice in most long-term care (LTC) facilities in the US. ${ }^{18}$ Self-assessment of health and QoL was performed with the Nottingham Health Profile (NHP) scale, which measures the subjective health state and HRQoL. ${ }^{19,20}$

The ADL scale assesses six daily living functions: bathing, getting (un)dressed, physiological needs, continence, eating and drinking, and mobility. The CDS questionnaire measures 15 human needs. This scale includes 15 items: eating and drinking, continence, body posture, mobility, day and night patterns, getting dressed and undressed, body temperature, hygiene, danger avoidance, communication, contact with others, sense of rules and values, daily activities, recreational activities, and learning abilities.

The IADL questionnaire is used to evaluate the complex activities of daily living. It examines the ability of senior citizens to cope in their own environment. The nine skills include: using the phone, walking to more remote places, the ability to shop and prepare meals, performing basic household chores (cleaning, minor repairs), unassisted taking of medications, and managing money. Each question has three possible answers: unassisted execution, execution with the assistance of other people, and a lack of independence. The maximum score is 27 .

The NHP questionnaire describes patients' emotional, social, and physical health problems. It is composed of two parts. The first part is concerned with fundamental problems in physical, psychological, and social functioning related to health state and consists of 38 questions in six subareas:

- energy level (EL): three questions

- pain (P): eight questions

- emotional reaction (ER): nine questions

- $\quad$ sleep (S): five questions

- social isolation (SI): five questions

- physical abilities (PA): eight questions.

The second part is concerned with the influence of actual health state on the seven spheres of life: work, housework, social life, everyday life, sexual life, hobbies and interests, and spending free time. Respondents had to answer 'yes' or 'no' on each question. The results of the research are estimated separately for each feature of the first part of the questionnaire. The higher the outcome, the worse the impairment of efficiency.

\section{Procedure and ethical considerations}

Approval for the study was obtained from the Bio-Ethical Committee at the university on the following conditions: the 
patients were chosen randomly; participation was voluntary; and all the patients received an oral invitation to participate in this research and signed an informed consent form.

This study was conducted at the Clinic of Geriatrics of Collegium Medicum in Bydgoszcz from October 2011 to December 2012, with the approval of the Ethics Committee at the university.

Permission to use the CDS questionnaire was given by Professor Ate Dijkstra. Permission to use the NHP questionnaire was given by Professor Kazimierz Wrzesniewski, who is the author of the Polish version.

\section{Statistical analysis}

The research results were subjected to statistical analysis using the STATISTICA program (v10; Dell Inc., Round Rock, TX, USA). The following methods of statistical analysis for countable data were used: 1) basic descriptive statistics including mean and standard deviation values; 2) the $\chi^{2}$ test - a method that performs a hypothesis to determine whether or not to reject the notion that two variables are independent; 3 ) Kramer's coefficient, which determines the strength of correlation; and 4) Student's $t$-test was used to calculate differences between means. A significance level of $P<0.05$ was used to determine statistical differences.

\section{Results}

The survey was conducted among 227 patients (149 women and $78 \mathrm{men})$. The mean age of the patients was 73.6 years, with 139 patients aged $60-74$ years $(61.2 \%), 79$ patients of $75-89$ years $(34.8 \%)$, and nine patients aged 90 years or over (4\%). Most of the patients lived in the city $(69.2 \%)$ and $30.8 \%$ in the countryside. Within the group, $5.7 \%$ had higher education, $34.4 \%$ secondary education, $26.4 \%$ elementary education, and $33.5 \%$ had followed a technical course. Most of the patients assessed their material status as good (85.9\%), $2.2 \%$ as average, and $6.6 \%$ as bad. As to marital status, 115 (50.7\%) were married, 102 (44.9\%) were widowed, and ten (4.4\%) declared their marital status as single.

Once the data had been collected, analysis of the functional performance of the patients was performed. The level of performance measured using the ADL was medium to high in most of the respondents. The efficiency of performance using the IADL in the study group was also at the medium to high levels of independence. However, quite a large percentage of respondents (40.1\%) showed low efficiency in performing the complex activities of daily living (Table 1).
Table I Results of ADL and IADL scores in the study group

n (\%) of respondents

\begin{tabular}{ll}
\hline ADL score classes & \\
High: $6.0-4.5$ & $139(61.2)$ \\
Medium: $4.5-2.5$ & $71(31.3)$ \\
Low: $\leq 2.5$ & $17(7.5)$ \\
Mean ADL: 4.5 & \\
IADL score classes & \\
High: $22-27$ & $87(38.3)$ \\
Medium: 2 I-16 & $49(21.6)$ \\
Low: $9-15$ & $91(40.1)$ \\
Mean IADL: 19.5 & \\
Total & $227(100)$
\end{tabular}

Abbreviations: ADL, Activities of Daily Living; IADL, Instrumental Activities of Daily Living.

For patients, the most important issues related to the fulfillment of needs were difficulties with the adoption of appropriate body posture/position, movement restrictions, and the problem of participating in leisure activities outside the home without assistance. Slightly less significant problems were related to maintaining good hygiene and performing daily activities. The patients coped best with the preparation and consumption of food, maintaining continence, communicating with others, and respecting values and principles. Other needs were met at the medium level. These results are shown in Table 2 .

The average CDS sum score was $61.1 \mathrm{p} ; 59.7 \mathrm{p}$ in men and $61.8 \mathrm{p}$ in women. The general deficit in fulfilling the needs of the subject group was low. Most of the patients were at the medium to high levels of the CDS scale ( $>45 \mathrm{p}$ ), remaining only partially dependent on nursing care (Table 3 ). The NHP scale was applied to all patients and showed that the biggest

Table 2 The items of CDS for the study group $(n=227)$

\begin{tabular}{|c|c|}
\hline Items of CDS & Mean \pm SD \\
\hline I. Eating and drinking & $4.3 \pm 1.0$ \\
\hline 2. Incontinence & $4.4 \pm 1.6$ \\
\hline 3. Body posture & $3.8 \pm 1.0$ \\
\hline 4. Mobility & $3.5 \pm 1.2$ \\
\hline 5. Day/night pattern & $4.4 \pm 0.8$ \\
\hline 6. Getting dressed and undressed & $4.1 \pm 1.0$ \\
\hline 7. Body temperature & $4.3 \pm 0.9$ \\
\hline 8. Hygiene & $3.9 \pm 1.0$ \\
\hline 9. Avoidance of danger & $4.0 \pm 0.8$ \\
\hline 10. Communication & $4.7 \pm 0.6$ \\
\hline II. Contact with others & $4.2 \pm 1.0$ \\
\hline 12. Sense of rules and values & $4.5 \pm 0.8$ \\
\hline 13. Daily activities & $3.9 \pm 1.1$ \\
\hline 14. Recreational activities & $3.2 \pm 1.3$ \\
\hline I5. Learning ability & $3.9 \pm 0.8$ \\
\hline
\end{tabular}

Abbreviations: CDS, Care Dependency Scale; SD, standard deviation. 
Table 3 Results of CDSSUM - scores in the study group

\begin{tabular}{ll}
\hline CDS score classes & $\mathbf{n}(\%)$ of respondents \\
\hline $\begin{array}{l}\text { I5-24 p: patient is completely } \\
\text { dependent }\end{array}$ & $0(0)$ \\
$\begin{array}{l}\text { 25-44 p: patient is to a great } \\
\text { extent dependent }\end{array}$ & $19(8.4)$ \\
$\begin{array}{l}\text { 45-59 p: patient is partially } \\
\text { dependent }\end{array}$ & $63(27.8)$ \\
$\begin{array}{l}\text { 60-69 p: patient is to a limited } \\
\text { extent dependent }\end{array}$ & $95(41.9)$ \\
70-76 p: patient is almost & $50(22)$ \\
independent & $61.1 \pm 10.1$ \\
CDSSUM - score, mean \pm SD
\end{tabular}

Abbreviations: CDS, Care Dependency Scale; p, points; CDSSUM, Care Dependency Scale-sum.

problems were related to sleep disturbances, in the form of difficulties in falling asleep and the need to use sleeping pills, followed by restrictions on free movement and the loss of vital energy. The patients felt permanent fatigue and a rapid loss of strength, and thus they performed various activities with great effort. Quite a large percentage of the patients complained of pain. Different spheres of life including housework, organization of free time and social life, as well as cultivating one's interests were affected by deteriorating health in elderly patients (Table 4). A significant difference between men and women in the section regarding pain was noted, with women feeling it more intensely $(P=0.01)$. The NHP questionnaire showed significant correlations involving pain in various diseases. Patients with disorders of the musculoskeletal system and cancer complained of pain significantly more often than other patients. Figure 1 shows this correlation. Patients who had suffered from their diseases for over 15 years experienced significantly greater pain than

Table 4 The items of NHP for the study group $(n=227)$

\begin{tabular}{ll}
\hline Items of NHP & Mean \pm SD \\
\hline I. Energy level & $52.4 \pm 36.1$ \\
2. Pain & $36.7 \pm 31.0$ \\
3. Emotional reaction & $31.6 \pm 27.7$ \\
4. Sleep & $58.9 \pm 34.3$ \\
5. Social isolation & $23.8 \pm 26.8$ \\
6. Physical abilities & $53.6 \pm 26.6$ \\
NHP - seven spheres of life & Yes/no \\
& $\mathbf{n}(\%)$ of respondents \\
I. Work & $73(32.2) / 154$ \\
2. Housework & $161(70.9) / 66$ \\
3. Social life & $119(52.4) / 108$ \\
4. Everyday life & $52(22.9) / 175$ \\
5. Sexual life & $99(43.6) / 128$ \\
6. Hobbies and interests & $120(52.9) / 107$ \\
7. Spending free time & $127(55.9) / 100$ \\
\hline
\end{tabular}

Abbreviations: NHP, Nottingham Health Profile; SD, standard deviation.

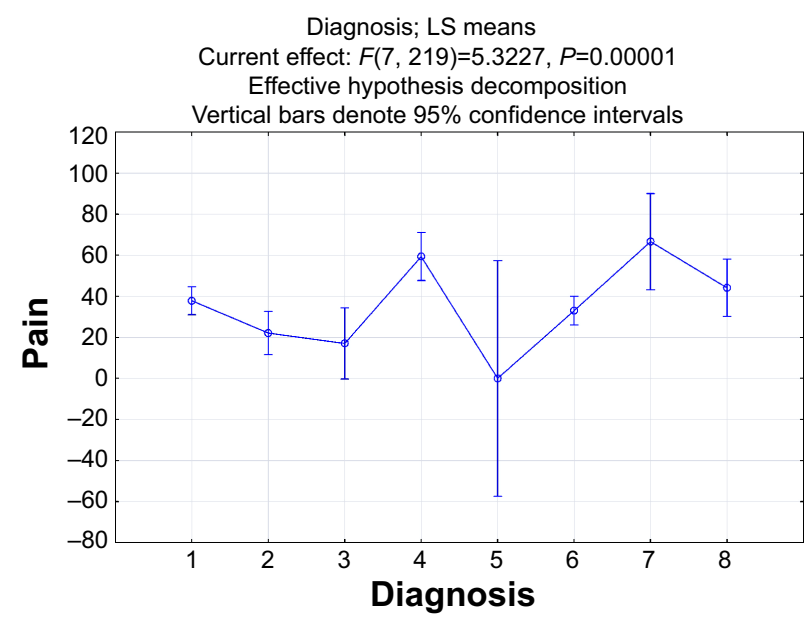

Figure I Pain depending on diagnosis.

Notes: I) Diseases of circulatory system; 2) diseases of respiratory system; 3 ) diseases of digestive system; 4) diseases of skeletal system; 5) diseases of urinary system; 6) polipathology; 7) oncological diseases; 8) other.

Abbreviation: LS, least squares.

those patients who had suffered for a shorter time (Figure 2). Patients with disorders of the musculoskeletal system had significantly greater movement limitations than patients with diabetes mellitus. Types and duration of disease did not have a material impact on the possibility of fulfilling biological, psychological, and social needs. Good daily functioning of elderly patients significantly depends on their intellectual and mental efficiency (Figure 3).

\section{Discussion}

In this study, the functional performance of patients was at a medium to high level, whereas the deficit in the fulfillment of their needs was low. Factors of particular concern for elderly

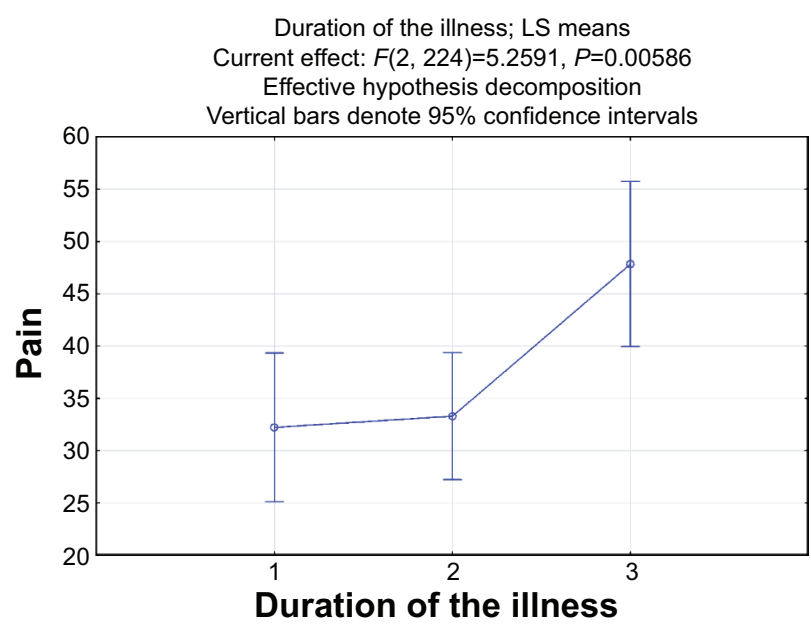

Figure 2 Pain depending on duration of illness in the study group. Notes: I) $<5$ years; 2 ) $5-15$ years; 3 ) $>15$ years.

Abbreviation: LS, least squares. 


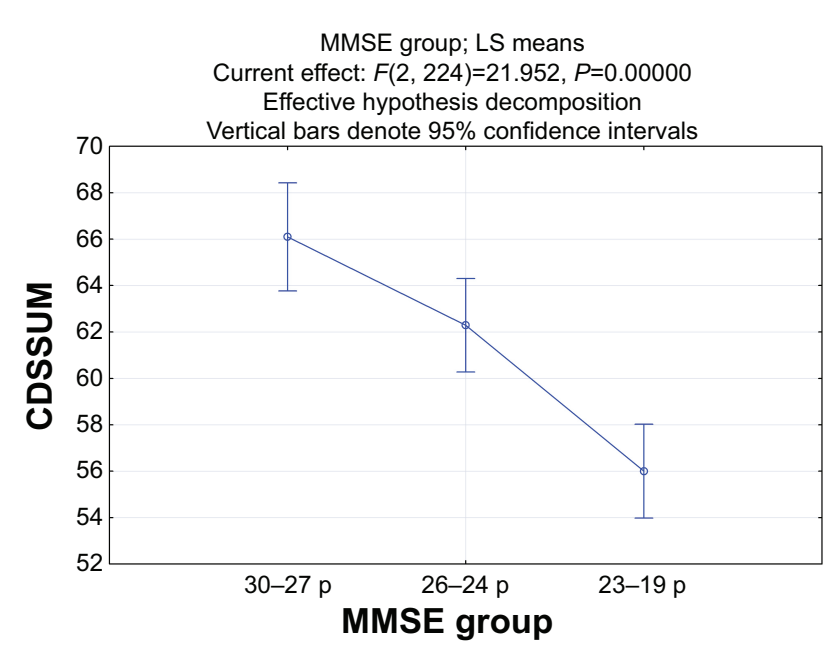

Figure 3 CDSSUM depending on MMSE in the study group.

Abbreviations: LS, least squares; MMSE, mini-mental state examination; p, points; CDSSUM, Care Dependency Scale-sum.

patients included sleep disturbances, limitations of mobility, loss of vital energy, and the presence of painful ailments. In chronically ill patients, the presence and intensification of problems in different areas of life mainly related to the performance of housework, organization of leisure time, and cultivating interests. The intensification of problems with pain was observed in women, as well as people suffering from movement disorders, cancer, or who had suffered from their diseases for longer periods.

Good daily functioning of elderly patients significantly depends on their intellectual and mental efficiency.

Our study showed that the mean CDS result of geriatric patients was $61.1 \mathrm{p}$, which indicates a medium degree of dependence on nursing care. The emerging issues were primarily related to the fulfillment of biological needs and difficulty in the organization of going out of the house. In comparison with previous studies, similar problems in the study group were observed. ${ }^{21}$ Lohrmann et al conducted studies in German nursing homes and hospitals, and dependence on nursing care was observed to increase with age and was related to the type of disease. However, the residents of nursing homes were completely dependent on, or substantially dependent on, nursing care, and they showed the largest deficits in the maintenance of personal hygiene, mobility, avoiding dangers, and daily activities. ${ }^{12}$ Our previous studies indicate that the HRQoL was related to age, sex, disease duration, place of living, education, treatment received, as well as some other factors. ${ }^{22}$ Current research has shown that QoL is related to sex; pain occurred more often in women and in patients with musculoskeletal system diseases and cancer. Restrictions of movement also concerned these patients significantly more often. Patients with cancer experienced the largest social isolation. Other social and demographic factors did not appear to play as important a role in this study. In our previous research related to geriatric patients with lower general functional capacity, we observed more nursing problems and higher deficiency in fulfilling needs in comparison to our present sample. ${ }^{23}$ Our current research was extended to include assessment of QoL, which required participants with higher functional capacity, especially in terms of intellectual capability. This might contribute to the variance between the current and previous samples, explaining the different results to some extent. The authors assume that good psychophysical status, functional capacity, adaptation to disease and limitations, and good health care and self-care in the study group, despite the chronicity of the illnesses, were important for fulfilling needs and prevent deterioration in QoL. A study conducted among the residents of nursing homes concluded that the NHP questionnaire can be used for patients with normal cognitive function or moderate cognitive impairment. ${ }^{24}$ It is important to have questions that are well-received and understood by patients, which make the assessment more objective assuming a minimal margin of error $(P<0.05)$. The authors of this manuscript describing the research tools for assessing the QoL among the elderly recognize the importance of the NHP questionnaire as one of the best tools for this age group. There are some studies conducted by other authors ${ }^{25}$ who used the NHP scale in people over 65 years showing a high level of cognitive performance (MMSE average: $26.1 \mathrm{p}$ ).

The results of the PolSenior research on health, social, psychological, and economic problems, conducted in the year 2010 in Poland in a representative sample of elderly people $(5,695$ persons), showed similarity to our research. Our clinic took part in the PolSenior population research of elderly people in Poland. The results were presented at national and international symposiums and are available on the website (www.iimcb.gov.pl). The authors have not encountered such an extensive dataset in Central and Eastern European countries. ${ }^{26}$

The analysis of the results on health issues showed that only a small number of the subjects were in good health. The majority had diseases/injuries, such as: diabetes mellitus, arterial hypertension and other circulatory disorders, falls, pain, and other medical problems. Patients admitted to our clinic had corresponding syndromes. ${ }^{27}$

As confirmed in this study, QoL represents a multidimensional concept that is based on subjective assessment of health by patients. Economic status, nutritional status, and functional and cognitive performance also influence the 
QoL. Distinguishing the subjective aspects of physical and mental health, and not just focusing on the biochemical and physiological parameters, has led to the development of this study. It is very important to assess the ability to function normally in society and in the family, to be free of various physical and mental ailments, as well as the economic and other problems associated with aging and the many diseases that usually need treatment. There are some studies pointing out good functioning of older people. This was determined by the significant cognitive performance of the studied group. Lower levels of cognitive function are associated with lower daily efficiency in elderly patients with a greater dependence on care.

\section{Conclusion}

This study indicates that health and care problems decrease QoL in older people. Furthermore, we confirm that elderly patients require a comprehensive, holistic approach to a variety of problems that occur with aging. Optimal care has to be individualized for each patient, with a personalized plan. Nurses prepared to care for geriatric patients can play an essential role in this task. It is worth expanding clinical research on aspects of QoL in future in order to optimize efficiency of increasing expenditure for the treatment and care of the elderly population.

\section{Acknowledgments}

We would like to thank the nurses and patients who voluntarily participated in the study.

\section{Author contributions}

Marta Muszalik contributed to the conception of the study and the study design, acquisition of data, statistical analysis and interpretation of data, draft of the paper, and giving final approval of the version to be published. Tomasz Kornatowski contributed to the study design, analysis and interpretation of data, drafting the article, and giving final approval of the version to be published. Halina Zielińska-Więczkowska contributed to the conception of the study and the study design, acquisition of data, statistical analysis and interpretation of data, draft of the article, and giving final approval of the version to be published. Kornelia Kędziora-Kornatowska contributed to the study design, analysis and interpretation of data, draft of the article and revision, and giving final approval of the version to be published. Ate Dijkstra contributed to the study design, analysis and interpretation of data, drafting of the article and revision, and giving final approval of the version to be published.

\section{Disclosure}

The authors report no conflicts of interest in this work.

\section{References}

1. Grodzicki T, Kocemba J, Skalska A. Geriatria z elementami gerontologii ogólnej. [Geriatrics with Elements of General Gerontology]. 1st ed. Gdańsk, Poland: Via Medica; 2006. Polish.

2. Kannan G, Rani V, Ananthanarayanan RM, et al. Assessment of quality of life of cancer patients in a tertiary care hospital of South India. J Cancer Res Ther. 2011;7(3):275-279.

3. Erceg P, Despotovic N, Milosevic DP, et al. Health-related quality of life in elderly patients hospitalized with chronic heart failure. Clin Interv Aging. 2013;8:1539-1546.

4. Taïeb D, Baumstarck-Barrau K, Sebag F, et al. Health-related quality of life in thyroid cancer patients following radioiodine ablation. Health Qual Life Outcomes. 2011;9:33.

5. Vest MT, Murphy TE, Araujo KL, Pisani MA. Disability in activities of daily living, depression, and quality of life among older medical ICU survivors: a prospective cohort study. Health Qual Life Outcomes. 2011;9:9.

6. Gunaydin R, Karatepe AG, Kaya T, Ulutas O. Determinants of quality of life (QoL) in elderly stroke patients: a short-term follow-up study. Arch Gerontol Geriatr. 2011;53(1):19-23.

7. Calero D, Navarro E. Differences in cognitive performance, level of dependency and quality of life (QoL), related to age and cognitive status in a sample of Spanish old adults under and over 80 years of age. Arch Gerontol Geriatr. 2011;53(3):292-297.

8. Oztürk A, Simşek TT, Yümin ET, Sertel M, Yümin M. The relationship between physical, functional performance and quality of life $(\mathrm{QoL})$ among elderly people with a chronic disease. Arch Gerontol Geriatr. 2011;53(3):278-283

9. De Walden Gałuszko K. The problem of quality of life in terminal patients. Medipress Palliative Med. 2003;1:4-6.

10. Dijkstra A. Care Dependency. An Assessment Instrument for Use in Long-Term Care Facilities [academic thesis]. 1st ed. Groningen, the Netherlands: University of Groningen; 1998.

11. Dijkstra A, Buist G, Moorer P, Dassen T. A reliability and utility study of the care dependency scale. Scand J Caring Sci. 2000;14: $155-161$.

12. Lohrmann Ch, Dijkstra A, Dassen T. The Care Dependency Scale: an assessment instrument for elderly patients in German hospitals. Geriatr Nurs. 2003;24(1):40-43.

13. Dijkstra A, Muszalik M, Kędziora-Kornatowska K, Kornatowski T. Care Dependency Scale - psychometric testing of the Polish version. Scand J Car Sci. 2010;24 Suppl 1:62-66.

14. Henderson V. The Nature of Nursing: A Definition and its Implications for Practice, Research and Education. 1st ed. New York, NY: MacMillan Press; 1966.

15. Abrams WB, Mark H, Beers MH, Berkow R. Medycyna geriatryczna. [Textbook of Geriatric Medicine]. 1st ed. Wrocław, Poland: Urban and Partner; 1999. Polish.

16. Katz S, Ford AB, Moskowitz R, Jackson BA, Jaffe MW. Studies of illness in the aged: The index of ADL, a standardized measure of biological and psychosocial function. JAMA. 1963;185:914-919.

17. Instrumental Activities of Daily Living (IADL) Scale. Original observer-rated version. "Does do" form - for women only. Psychopharmacol Bull. 1998;24(4):785-787.

18. Clifford AP, Cipher DJ, Roper KD. Assessing resistance to activities of daily living in long-term care. J Am Med Dir Assoc. 2003;4(6): 313-319.

19. Hunt SM, McKenna SP, McEwen J, Williams J, Papp E. The Nottingham Health Profile: subjective health status and medical consultations. Soc Sci Med A. 1981;15(3 Pt 1):221-229.

20. Wrzesniewski K. Development of a Polish version of the Nottingham Health Profile. Quality of Life Newsletter. MAPI Research Institute. 2000;25:10. 
21. Muszalik M, Dijkstra A, Kędziora-Kornatowska K, ZielińskaWięczkowska $H$. Health and nursing problems of elderly patients related to bio-psycho-social need deficiencies and functional assessment. Arch Gerontol Geriatr. 2012;55(1):190-194.

22. Muszalik M, Bartuzi Z, Kędziora-Kornatowska K, Marzec A. The Nottingham Health Profile research of subjective state of health based on the Polish adaptation of the NHP. Ann Univ Med M Curie-Sklodowska. 2006;60(483):90-94.

23. Muszalik M, Kędziora-Kornatowska K, Kornatowski T. Functional assessment and health-related quality of life (HRQOL) of elderly patients on the basis of the functional assessment of chronic illness therapy (FACIT)-F questionnaire. Arch Gerontol Geriatr. 2009;49(3):404-408.

24. Tabali M, Jeschke E, Dassen T, Ostermann T, Heinze C. The Nottingham Health Profile: a feasible questionnaire for nursing home residents? Int Psychogeriatr. 2012;24(3):416-424.
25. Kostka T, Jachimowicz V. Relationship of quality of life to dispositional optimism, health locus of control and self-efficacy in older subjects living in different environments. Qual Life Res. 2010;19(3):351-361.

26. Bledowski P, Mossakowska M, Chudek J, et al. Medical, psychological and socioeconomic aspects of aging in Poland: assumptions and objectives of the PolSenior project. Exp Gerontol. 2011;46(12):1003-1009.

27. Mossakowska M, Wiecek A, Blędowski P. Medyczne, psychologiczne, socjologiczne i ekonomiczne aspekty starzenia w Polsce. [Medical, psychological, sociological, and economic aspects of ageing in Poland]. Poznań, Poland: Termedia; 2012. Polish.
Clinical Interventions in Aging

\section{Publish your work in this journal}

Clinical Interventions in Aging is an international, peer-reviewed journal focusing on evidence-based reports on the value or lack thereof of treatments intended to prevent or delay the onset of maladaptive correlates of aging in human beings. This journal is indexed on PubMed Central, MedLine,

\section{Dovepress}

CAS, Scopus and the Elsevier Bibliographic databases. The manuscript management system is completely online and includes a very quick and fair peer-review system, which is all easy to use. Visit http://www.dovepress. com/testimonials.php to read real quotes from published authors. 